\title{
Detecção e Hierarquização de Conglomerados de Focos de Aedes aegypti pela Estatística Scan com um Modelo de Permutação Espaço-temporal
}

\section{Detection and Hierarchy of Aedes aegypti Clusters by the Scan Statistic with a Space-time Permutation Model}

Ivan Merêncio ${ }^{1}$ e Carlos Antonio Oliveira Vieira ${ }^{2}$

1 Universidade Federal de Santa Catarina, Programa de Pós-Graduação em Engenharia de Transportes e Gestão Territorial, Florianópolis, Brasil. ivan.merencio@posgrad.ufsc.br.

ORCID: https://orcid.org/0000-0003-1288-124X

2 Universidade Federal de Santa Catarina, Departamento de Geociências, Programa de Pós-Graduação em Engenharia de Transportes e Gestão Territorial, Florianópolis, Brasil. carlos.vieira@ufsc.br.

ORCID: https://orcid.org/0000-0003-4047-8466

Resumo: A utilização da estatística espacial associada ao estudo de dados epidemiológicos humanos intensificou-se nas últimas duas décadas. Nesse sentido, este artigo aborda a estatística de varredura (estatística Scan) para a detecção de conglomerados espaço-temporais de focos de Aedes aegypti na zona urbana de Joinville, Santa Catarina. O controle deste vetor é de grande preocupação para as cidades brasileiras, as quais tem dificuldades para conter a infestação, devido ao modo de sobrevivência do mosquito e os diversos fatores relacionados à sociedade. O objetivo deste estudo é aplicar um modelo estatístico espaço-temporal para a identificação de aglomerados, definindo sua localização, dimensão e ordem. A sistematização da base de dados abrangeu os registros dos focos diários de Ae. Aegypti, do período de janeiro de 2009 a setembro de 2018, fornecidos pela Diretoria de Vigilância Epidemiológica (DIVE) de Santa Catarina. Os focos foram georreferenciados com o auxílio dos dados cadastrais de Joinville. Para a execução da estatística considerou-se o modelo de probabilidade permutação espaço-temporal, apoiado na abordagem retrospectiva. No resultado da pesquisa evidenciou-se a capacidade do procedimento para delimitação de clusters históricos e ativos, sendo que o modo retrospectivo identificou 26 agrupamentos; o único ativo situou-se no bairro Boa Vista, o que comprova que a região necessita de medidas de intervenção. O desenvolvimento do método demonstrou-se eficiente para o conhecimento de clusters históricos e, principalmente, para a definição de surtos precoces. Isso caracteriza a estatística Scan como uma ferramenta importante e de baixo custo para orientar a vigilância ambiental nas atividades de controle do inseto.

Palavras-chave: Estatística scan. Análise retrospectiva. Cartografia e saúde pública.

\begin{abstract}
The use of spatial statistics associated with human epidemiological studies has intensified in the last two decades. In this sense, this article uses scan statistic for detections of spatio-temporal clusters of Aedes aegypti outbreaks at the urban area of Joinville, Santa Catarina. The control of this insect is very important for many cities in Brazil, which has presented some difficult in order to restring infestations, due to the survival of these insects and also dangerous factors related to the society. The aim of this paper is to apply an statistic spatio-temporal model in order to identify clusters, defining its location, size and order. The systematization of the database covers the dairy records of Ae. aegypti outbreaks, of the period from January 2009 to September 2018, led by the Office of Epidemiological Surveillance of Santa Catarina. The outbreaks which were located with the use of the cadastral data of Joinville. For the statistical analysis, the space-time probability model was used, based on the retrospective approach. In the result of this research it was possible to showed the potential of this model in order to detect historical and active clusters. The retrospective approach has identified 26 clusters; the only one active was located at the Boa Vista neighborhood, which shows that this region needs intervention measures. The development of the method was efficient for knowledge of historical clusters and, mainly, for definition of early outbreaks. This characterizes Scan statistics as an important tool in order to guide the sanitary activities for insect control.
\end{abstract}

Keywords: Scan Statistics. Retrospective analysis. Cartography and public health.

\section{INTRODUÇÃO}

As primeiras ações destinadas à prevenção e ao combate do Aedes aegypti no Brasil foram realizadas em 1942. Nessa época, a Organização Pan-Americana da Saúde (OPAS) organizou um programa de controle 
vetorial voltado para a eliminação dos focos de febre amarela urbana. Assim, entre 1958 e 1965, o Brasil e mais dezessete países da América Latina receberam o certificado de erradicação do Ae. aegypti e a transmissão de dengue foi interrompida, porém o programa foi extinto em meados de 1970 e, consequentemente, a reintrodução do mosquito no país ocorreu em 1976 (DICK et al., 2012).

De maneira geral, nota-se a preocupação dos países em desenvolvimento para conter as populações de insetos vetores, embora nas duas últimas décadas tenha ocorrido o aumento da incidência de arboviroses, protozooses e helmintoses, principalmente nos países tropicais e subtropicais (OPAS, 2010). O Ae. aegypti é o principal transmissor das doenças: dengue, chikungunya, zika e febre amarela urbana (KRAEMER et al., 2015). O Brasil detém elevado número de casos de dengue e é um dos países com a maior taxa no mundo. No período de 1990 a 2016 foram identificados mais de 12 milhões de infectados pela doença no país, com aproximadamente 1,5 milhões somente no ano de 2016 (BRASIL, 2017).

Em Santa Catarina (SC) a quantidade de casos autóctones dessas arboviroses é baixa (SANTA CATARINA, 2017a). Ainda assim, a contagem de focos do mosquito indica a sua elevada proliferação no espaço urbano, resultando assim no aumento do número de cidades consideradas infestadas, categoria a qual se encontra o município de Joinville (SANTA CATARINA, 2017b). A situação demanda atenção da sociedade, já que um surto epidêmico poderia gerar grandes prejuízos.

Em função da dificuldade de se conter o avanço da infestação, diversos estudos têm sido realizados na tentativa de inserir novas tecnologias e métodos para que a redução dos números do inseto seja bem-sucedida. Nesse cenário, pode-se salientar a utilização da estatística scan (ou de varredura), originalmente apresentada por Kulldorff et al. (2005), que visa avaliar estatisticamente o objeto de estudo no espaço geográfico para apoiar o processo de tomada de decisão e assim evitar uma possível epidemia. Há também fatores que fazem com que as secretarias de vigilância priorizem o monitoramento e prevenção de alguns locais em detrimento de outros. Nessa perspectiva, a técnica de detecção e hierarquização de clusters indica ser um recurso importante para a identificação das áreas que demandam maior atenção (ARAÚJO, 2012).

Considerando a situação atual da infestação na cidade de Joinville, este artigo tem como objetivo aplicar a Estatística Scan para a detecção de agrupamentos de Ae. aegypti no perímetro urbano de Joinville ao nível de significância de 5\%, classificando-os conforme a prioridade de intervenção, possibilitando assim o estudo da evolução espaço-temporal do vetor na cidade. Para isso, utilizou-se a série de dados de focos do mosquito no período de janeiro de 2009 a setembro de 2018 na área urbana do munícipio, fornecida pela Diretoria de Vigilância Epidemiológica de Santa Catarina (DIVE-SC) da Secretaria Estadual de Saúde, associada aos dados do cadastro territorial multifinalitário (CTM), disponibilizados no Sistema de Informações Municipais Georreferenciadas (SIMGEO) da Prefeitura de Joinville.

Desse modo, a detecção e hierarquização de clusters espaço-temporais significativos, com a representação dos resultados através de tabelas e cartas, demonstrou-se útil para a identificação de locais prioritários de intervenção, ou seja, uma ferramenta vantajosa para auxiliar o planejamento e execução de ações sanitárias de acordo com as características locais, aumentando a eficiência de combate ao inseto.

Este artigo está organizado da seguinte maneira: a Seção 2 apresenta uma breve teoria sobre a estatística Scan, englobando o método abordado nesta pesquisa; na Seção 3 delimita-se a área de estudo, os dados e os procedimentos metodológicos utilizados. Resultados e discussões do experimento realizado são apresentados na Seção 4; por último, considerações finais e perspectivas futuras são ponderadas na Seção 5.

\section{ESTATÍSTICA DE VARREDURA: MODELO DE PERMUTAÇÃo ESPAÇO- TEMPORAL}

A estatística de varredura procura identificar uma região composta por aglomerados, relacionando o número de casos observados em relação aos esperados. Esses clusters são constituídos alterando o raio, que varia até um valor máximo, de modo que não inclua mais de $50 \%$ da população em seu interior. Para cada circunferência ou cilindro (análise espaço-temporal) calcula-se a razão entre a verossimilhança com a hipótese alternativa de que o risco de ocorrência do evento é maior dentro do que fora do cilindro. A hipótese nula é de que a diferença entre as duas verossimilhanças seja ao acaso (KULLDORFF; NAGARWALLA, 1995; KAQUI, 2016). 
Pode-se definir que: $N$ é o número total de indivíduos na população, $C$ é o número total de casos observados, $Z$ é o conjunto das $z$ áreas candidatas a formarem um aglomerado. Estes $z$ candidatos são círculos de raios $r$ arbitrários situados em um centroide (Figura 1). Os círculos são limitados ao raio que estipula que o agrupamento $z$ contenha no máximo 50\% da população total da área (KULLDORFF, 1997).

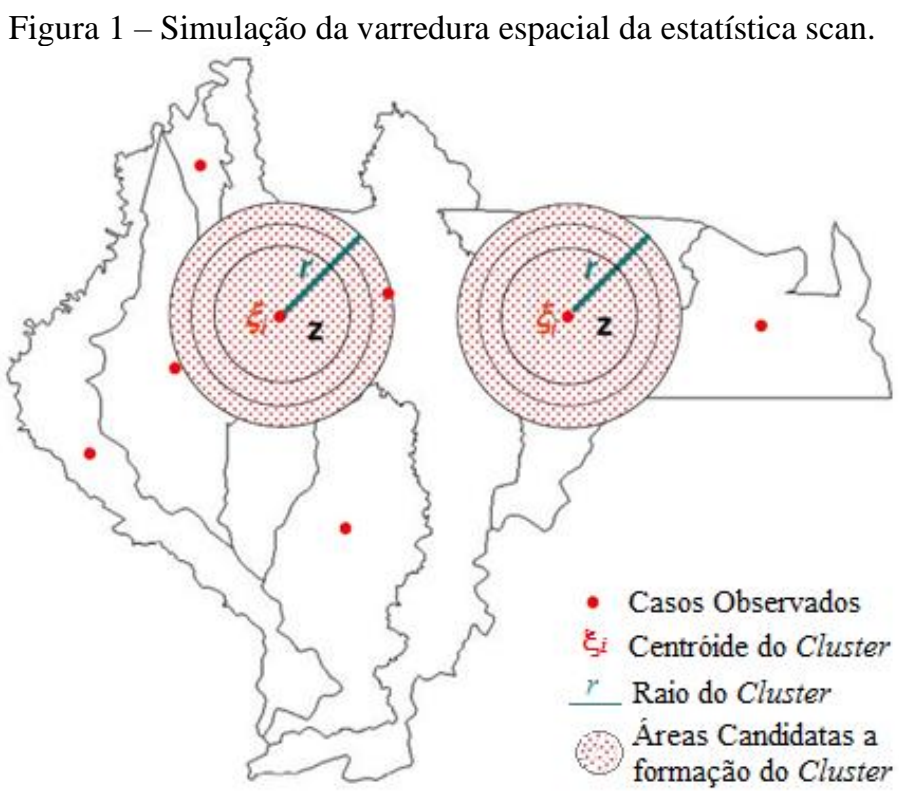

Fonte: Adaptado de Balieiro (2008).

Existem duas análises que podem ser executadas em dados puramente temporais e espaço-temporal, a análise retrospectiva e a prospectiva. A primeira preocupa-se com a detecção, em um espaço e intervalo fixados, de conglomerados históricos, que deixaram de ocorrer antes do término do estudo, e clusters ativos, que existirão até o fim da pesquisa. A última foca apenas na determinação dos aglomerados ativos. Nesse caso, a varredura é repetida em um período definido, por exemplo, um dia, um mês, um ano; o processo analisa os intervalos de tempo sucessivamente, considerando a informação do primeiro até a última data disponível (SILVA, 2016).

O modelo espaço-temporal torna o plano em três dimensões (Figura 2). O raio do cilindro corresponde a varredura na componente espacial e a altura representa a variação do tempo (BALIEIRO et al., 2009).

Figura 2 - Esquema de funcionamento da varredura espaço-temporal.

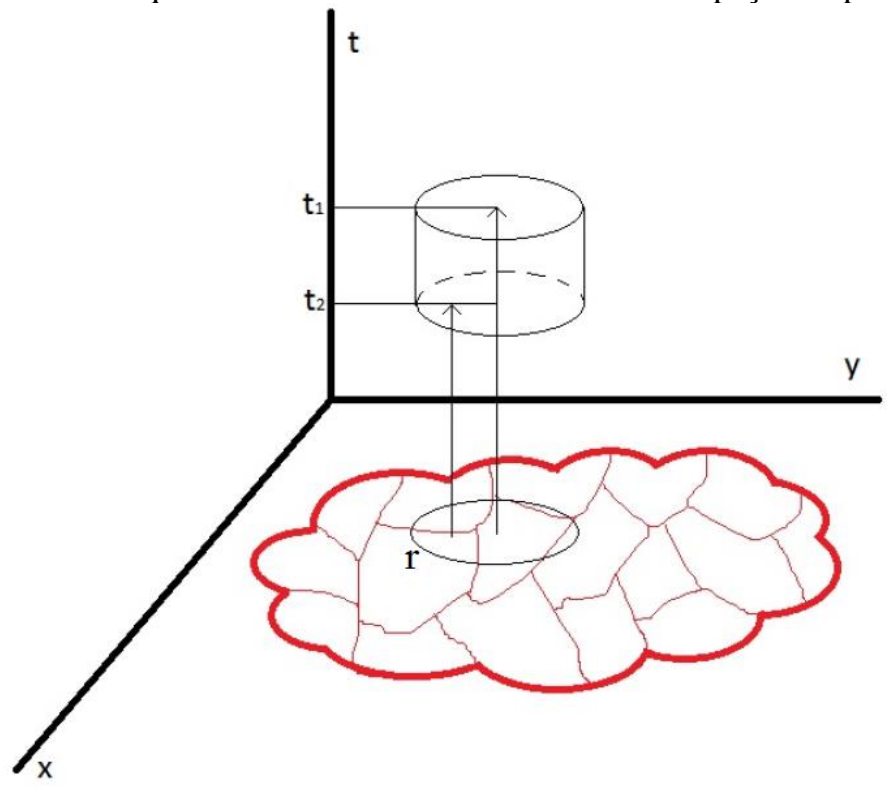

Fonte: Adaptado de Silva (2016). 
Na comparação entre os métodos Estatística Scan, Besag-Newell e Modelos Aditivos Generalizados (MAG) no estudo espacial de crimes em Houston (EUA) em 2005, a Estatística Scan demonstrou ser mais útil do que as demais ferramentas disponíveis para a análise, devido a maior disponibilidade de modelos de probabilidade e sua capacidade de adaptação (co-variáveis e análise espaço-temporal), evitando que a configuração de parâmetros fosse subjetiva (HELBICH; LEITNER, 2012). Quando confrontado com o Mapeamento Bayesiano de Doenças (MBD) na detecção de leishmaniose tegumentar em unidades do exército iraniano entre 2014 e 2017, o seu desempenho foi semelhante ao MBD (AYUBI et al., 2018).

Neste artigo, optou-se por detalhar somente o modelo de probabilidade de permutação espaçotemporal, para mais informações sobre os outros modelos consultar Kulldorff (2018) e Jung et al. (2010).

O modelo de permutação espaço-temporal é recomendado para estudos em que a base de dados é constituída com no mínimo: número de casos, localização espacial (coordenadas) e a data de ocorrência. Não necessita de informações de controle, tampouco sobre a população em risco (KULLDORFF, 2018).

Este modelo tem as seguintes características: sua varredura é feita no espaço e no tempo simultaneamente, isto é, não busca conglomerado espacial ou temporal de modo isolado; possibilita a realização do estudo apenas com os casos; na confirmação da hipótese nula, o seu modelo probabilístico indica que esses eventos seguem a distribuição hipergeométrica (BALIEIRO, 2008).

A verossimilhança é calculada para cada cilindro levando em conta o número de pontos dentro e fora dele com a quantidade de eventos esperados, cujo aglomerado com a máxima Razão de Verossimilhança Generalizada de Poisson (RVG) e que exceda o limite de casos esperados, é apontado como o agrupamento mais verossímil, sendo o principal candidato a um verdadeiro surto (KULLDORFF et al., 1998; GOMES, 2016).

O teste de significância é gerado a partir de várias permutações aleatórias dos casos no espaço e no tempo, relacionando-as com a localização inicial, garantindo que as componentes espacial e temporal se mantenham intactas. Em seguida, calcula-se a RVG para cada conjunto simulado. A significância da estatística é feita pelo teste de Monte Carlo, cuja hipótese nula da inexistência de aglomerados é rejeitada ao nível de 0,05 quando o p-valor simulado for menor ou igual a esse valor para o agrupamento mais verossímil (MATEUS, 2013).

\section{MATERIAIS E MÉTODOS}

\subsection{Caracterização da área de estudo}

O município de Joinville está localizado na região sul do Brasil, na mesorregião norte catarinense (Figura 3). É a maior cidade do estado de Santa Catarina, com uma população estimada em 577.077 habitantes, distribuídos ao longo de sua área de aproximadamente 1.126,10 $\mathrm{Km}^{2}$ (IBGE, 2017). A sede municipal tem como coordenadas geográficas: latitude $26^{\circ} 18^{\prime} 05^{\prime \prime}$ S e longitude $48^{\circ} 50^{\prime} 38^{\prime \prime}$ W, Sistema de Referência horizontal SIRGAS 2000. Está distante $180 \mathrm{~km}$ da capital Florianópolis.

Seu relevo é caracterizado pela presença de montanhas, planalto e planície. O ponto mais alto é o pico de Joinville com altitude de $1.350 \mathrm{~m}$ (OLIVEIRA, 2006). A Serra do Mar e o mangue são obstáculos a expansão da cidade, que resultam em um traçado urbano no sentindo norte-sul, acompanhando a rodovia BR101 e a baía da Babitonga (SANTANA, 1998).

O clima predominante da região é do tipo "mesotérmico, úmido, sem estação seca", conforme a classificação de Köppen. A umidade relativa média anual do ar é de 76,04\%. (JOINVILLE, 2017).

As maiores temperaturas para a cidade ocorrem nos primeiros meses do ano, cujas médias mensais são superiores a $25^{\circ} \mathrm{C}$, tendo maior destaque o mês de fevereiro. Já as temperaturas mais baixas estão relacionadas aos meses de junho até setembro, com médias mensais inferiores a $20^{\circ} \mathrm{C}$. Entretanto, entre maio a junho podem ocorrer dias em que as temperaturas máximas absolutas ultrapassem $30^{\circ} \mathrm{C}$ (JOINVILLE, 2016).

O município apresenta os maiores totais de precipitação do estado. O regime de chuvas anual varia entre $1.500 \mathrm{~mm}$ a $2.000 \mathrm{~mm}$, com os maiores índices de pluviosidade registrados nas estações da primavera e do verão. No entanto, há uma redução desse indicador no inverno (BARBOSA, 2009).

A vegetação predominante na região é resultado da ação combinada de fatores como geologia, clima, 
cobertura vegetal e hidrografia, o que faz com que a floresta ombrófila densa e os manguezais sejam os tipos dominantes. O mangue ocorre nas margens da lagoa do Saguaçu e da Baía da Babitonga, com cerca de 36,54 $\mathrm{km}^{2}$, mais de 50\% da área total da baía (JOINVILLE, 2017).

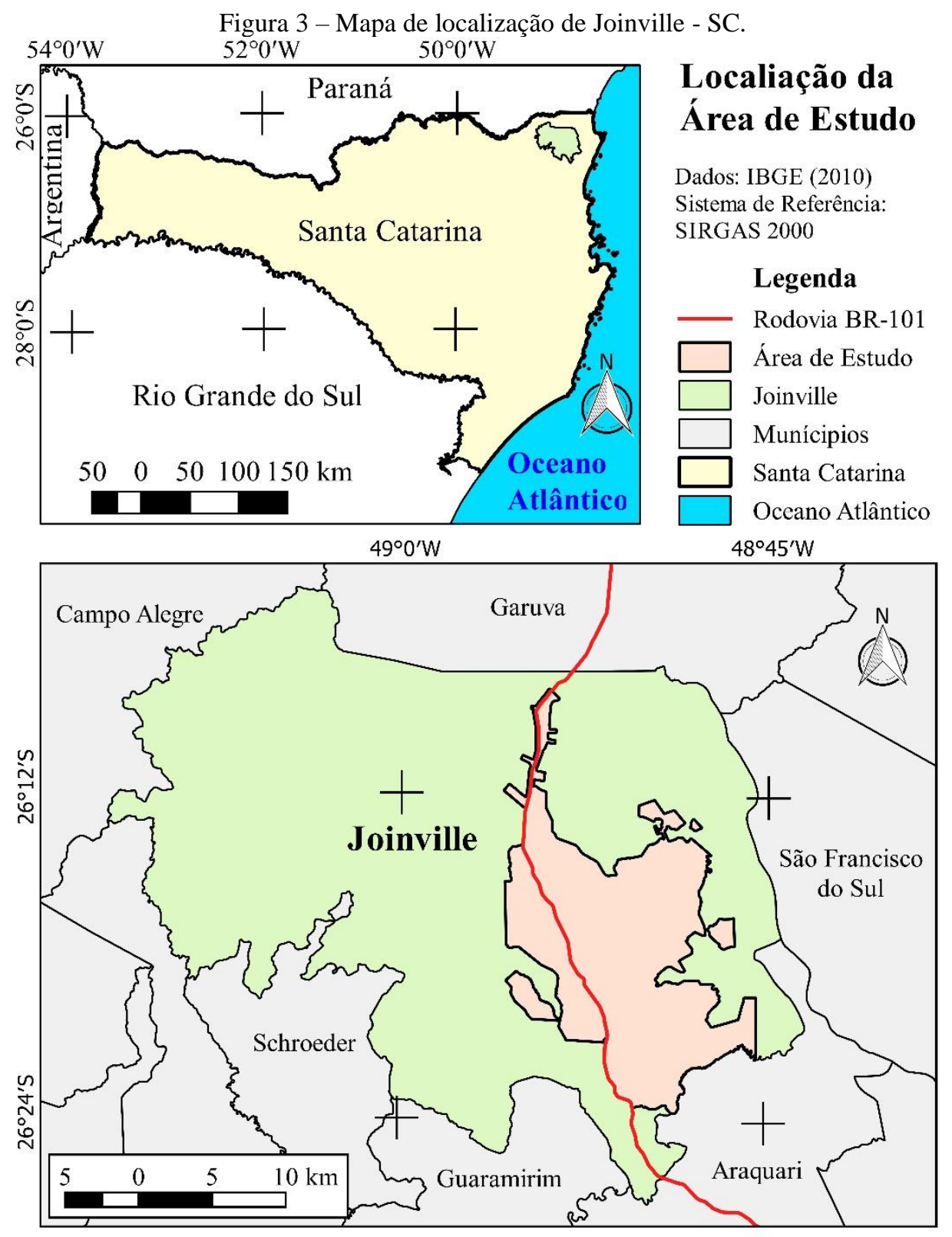

Fonte: Os Autores (2021).

\subsection{Base de dados}

A base cadastral foi obtida no Sistema SIMGEO da Prefeitura de Joinville, contendo ruas, lotes, edificações, quadras, limites de bairros, número da edificação e nome do logradouro. Esses dados são oriundos da atualização cadastral realizada em 2010 e estavam no formato Shapefile, Sistema de Referência Horizontal SIRGAS 2000 e coordenadas geodésicas.

Os relatórios contendo o número de focos de Ae. aegypti do período de janeiro de 2009 a setembro de 2018 em Joinville foram disponibilizados pela DIVE-SC. Os documentos possuíam informações sobre os focos, como: identificação, data da coleta, endereço, tipo do criadouro, ocupação do solo, entre outros (Tabela 1). 
Tabela 1 - Exemplo de atributos descritivos dos focos de Ae. aegypti em 2016 oriundos da base descritiva da DIVE-SC. ${ }^{1}$ ARM: Armadilha. ${ }^{2}$ PE: Ponto Estratégico.

\begin{tabular}{c|c|c|c|c|c}
\hline Número do Foco & Localidade & Imóvel & Data da Coleta & Formas Aquáticas & Ovos \\
\hline ARM $^{1}$ 2/16 & Floresta & Comércio & $06 / 01 / 2016$ & 5 & 0 \\
PE $^{2} 3 / 16$ & Itaum & Residência & $07 / 01 / 2016$ & 1 & 0 \\
ARM 4/16 & João Costa & Comércio & $13 / 01 / 2016$ & 11 & 0 \\
ARM 8/16 & Itaum & Comércio & $14 / 01 / 2016$ & 22 & 0 \\
\hline
\end{tabular}

Fonte: Adaptado de DIVE - SC (2018).

Essas planilhas eletrônicas contendo os dados descritivos foram manipuladas e formatadas no Software LibreOffice Calc. O Google Earth Pro, na versão gratuita, foi utilizado para identificar a localização dos focos do mosquito. Posteriormente, foi possível compilar os atributos descritivos com a base cadastral no Quantum GIS 2.18.9. Por fim, empregou-se o SATSCAN 9.6, embasado no modelo de permutação espaço-temporal, para aplicação da estatística de varredura

\subsection{Método}

A sequência das etapas de aplicação e desenvolvimento do método de detecção de conglomerados de Ae. aegypti, contendo a sistematização dos dados, assim como a escolha do modelo de probabilidade, a definição dos parâmetros estatísticos e a aplicação da estatística scan são apresentadas a partir do item 3.3.1 e resumidas no fluxograma da Figura 4.

Figura 4 - Fluxograma com as etapas de desenvolvimento do método.

Dados de Entrada

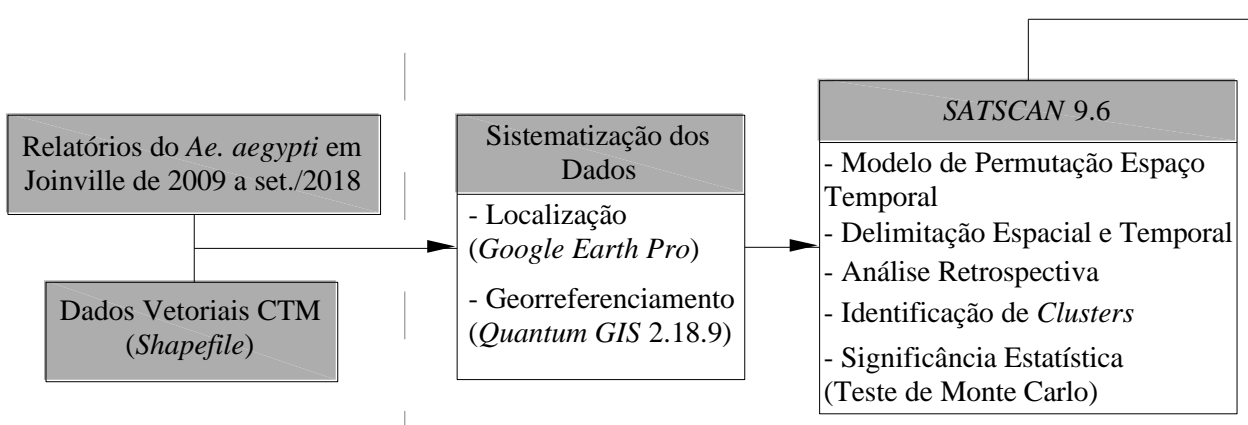

Dados de Saída

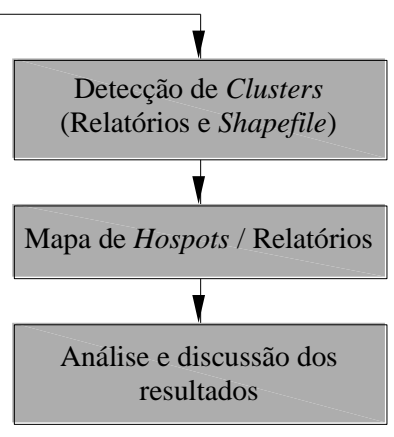

Fonte: Os autores (2021).

O georreferenciamento dos focos do vetor, iniciou com uma pré-localização no Google Earth Pro e posteriormente com a adequação ao interior dos lotes no Quantum GIS 2.18.9, que continha a base cadastral do distrito sede de Joinville configurada com o sistema de coordenadas geodésicas e Sistema de Referência horizontal SIRGAS2000. No software LibreOffice Calc foi relacionado as coordenadas dos criadouros aos atributos descritivos.

Para verificar a existência de conglomerados optou-se pelo modelo de permutação espaço-temporal (KULLDORFF et al., 2005), pois os relatórios eram compostos de uma contagem de focos do inseto e não havia informação para determinar com precisão qual a população em risco. Ademais, sua varredura é feita no espaço e no tempo simultaneamente, que é o diferencial desse método e essencial para que o objetivo deste trabalho fosse auferido. Não foi possível o uso dos modelos exponencial e normal pelo fato dos focos não serem uma variável contínua. Como o objetivo da pesquisa era relacionado à contagem de criadouros, a utilização do modelo ordinal não era adequada. Nesse caso, a variável resposta foi o número de focos do vetor identificados, que são dados pontuais com centroides localizados no interior dos lotes, no período de janeiro de 2009 a setembro de 2018 no município de Joinville.

A estatística scan trabalha com janelas de varredura com formato circular para diversos raios de busca, portanto foi imprescindível a definição desse limite. Assim, estipulou-se como valor máximo para o raio de 
busca 0,50 quilômetros, baseado no estudo de Freitas e Oliveira (2009). As variáveis climáticas de Joinville e a quantidade de focos indicam que a fêmea dificilmente encontrará condições adversas para que realize um deslocamento máximo a partir do ponto de eclosão em busca de ambiente favorável.

Além disso, a escolha pelo modelo de permutação espaço-temporal fez com que houvesse necessidade de informar o limite de busca no tempo. Embora ocorra sazonalidade no desenvolvimento do transmissor, informada por Ribeiro et al. (2015) e Busato et al. (2014), definiu-se como limite temporal máximo a porcentagem de $50 \%$ das ocorrências no interior do cluster, com o intuito de reconhecer a data de início da infestação e se os aglomerados cessariam sua atividade conforme a sazonalidade.

Em seguida, no ambiente do SATSCAN 9.6 realizou-se a busca de conglomerados baseado na análise retrospectiva, que identifica a presença tanto de clusters ativos quanto de históricos, isto é, que deixaram de existir antes do término da pesquisa.

Para cada suposto aglomerado foi calculado o valor da RVG. Aquele que apresentasse a maior grandeza era o mais provável, denominado primário. Para o teste da significância desse conglomerado primário considerou-se $5 \%$ de probabilidade, com as seguintes hipóteses:

HO: não existe agrupamento espaço-temporal dos focos de Ae. aegypti em Joinville;

H1: existe agrupamento espaço-temporal dos focos de Ae. aegypti em Joinville.

O procedimento de Monte Carlo foi utilizado para testar essas hipóteses com relação ao cluster primário. Se após as 999 permutações a ordem $R$ estivesse acima de 950 , contatava-se que o agrupamento do número de focos naquela zona não aconteceu devido ao acaso. Os conglomerados com valores de RVG menores, ditos secundários, tiveram avaliação da significância de modo similar ao realizado no primário.

Por fim, utilizou-se o software QuantumGIS 2.18.9 com a base cartográfica para espacialização dos resultados obtidos no SATSCAN 9.6, finalizando-os com a criação da carta para o munícipio de Joinville, na qual consta os círculos que correspondem ao diâmetro dos conglomerados detectados.

\section{RESULTADOS E DISCUSSÃO}

A cidade de Joinville registrou entre janeiro de 2009 e setembro de 2018 um total de 1.846 focos do inseto, entre formas aquáticas e adultas. Sendo que o intervalo dos últimos cincos anos (2014-2018) correspondeu com 78,33\% das notificações. O ano de 2009 apresentou 42 focos do vetor (2,27\%), que foi a menor quantidade para o período analisado, já o intervalo temporal entre os meses de janeiro a setembro de 2018 conteve a maior, 516 criadouros $(27,95 \%)$.

Agrupado os dados por semestre entre abril a setembro do mesmo ano e outubro a março do ano subsequente (Gráfico 1) observou-se a sazonalidade no índice de infestação, já que na maioria dos intervalos entre abril a setembro houve redução da ocorrência dos focos. Só aconteceram dois momentos em que essa situação foi atípica. No outono e inverno de 2014 o número de notificações superou o semestre anterior (primavera-verão), passando de 79 para 91 e, consequentemente na janela de 2014-2015 houve um aumento expressivo para 271 registros do inseto. A outra ocasião foi no semestre de 2017-2018 em que foi identificado o maior surto do vetor na série analisada, 301 criadouros, que declinaram para 295 no outono e inverno de 2018, ou seja, diminuição de apenas 6 casos.

A estatística scan identificou 26 conglomerados significativos ( $\mathrm{p}<0,05)$ (Tabela 2). O cluster primário (P1) foi detectado no bairro Itaum com uma RVG de 174,01, um raio de 0,50 Km e centroide com latitude $26,333434^{\circ} \mathrm{S}$ e longitude $48,839770^{\circ} \mathrm{W}$. Nessa área, no prazo de 15 meses ocorreram 292 focos de Ae. aegypti. Sob a hipótese nula, de que não existisse agrupamento, seriam esperados somente 81,95 casos. O $p$-valor para este aglomerado, obtido a partir de 999 simulações pelo método de Monte Carlo, foi igual a 1x10-17. Como o valor resultante é menor que 0,05 a hipótese nula da não existência do cluster primário foi rejeitada, o que indica que o valor foi significativo. Em relação a sua situação, trata-se de um agrupamento histórico, ou seja, não-ativo, pois ocorreu de 25/12/2013 até 24/03/2015, não permanecendo em atividade até o fim do estudo. O estabelecimento da significância dos conglomerados secundários seguiu o mesmo procedimento. Os resultados foram espacializados através da Carta dos aglomerados de Ae. aegypti detectados pela análise retrospectiva em Joinville-SC no período de janeiro de 2009 a setembro de 2018 (Figura 5). 
Gráfico 1 - Distribuição semestral dos focos de Ae. aegypti em Joinville - SC de abril de 2009 a setembro de 2018.

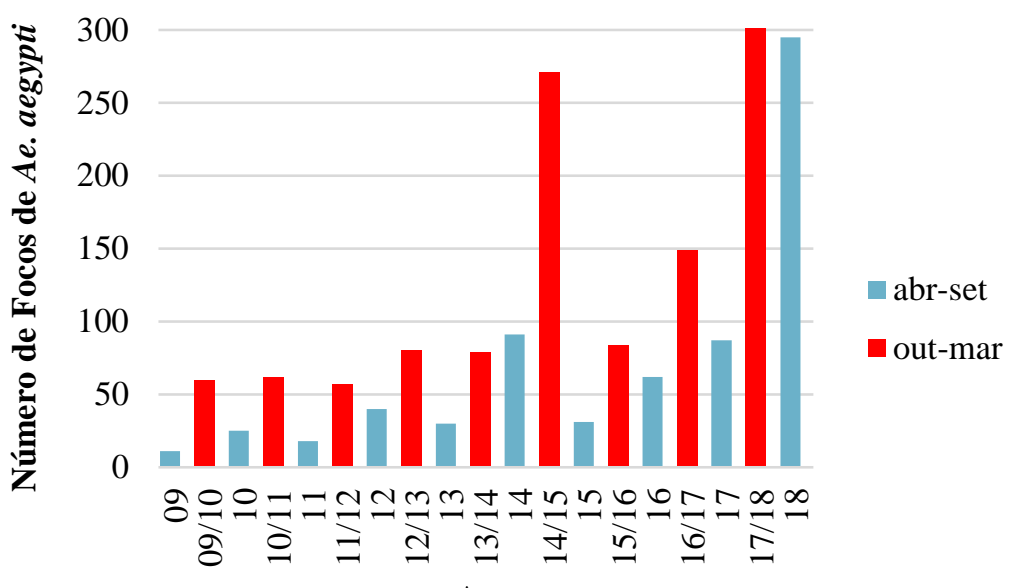

Ano

Fonte: Os autores (2021).

Tabela 2 - Aglomerados dos focos de Ae. aegypti detectados em Joinville-SC no período de janeiro de 2009 a setembro

\begin{tabular}{|c|c|c|c|c|c|c|c|}
\hline Cluster & Centroide / Raio (Km) & De & Até & Focos Obs. & Focos Esp. & RVG & $p$-valor \\
\hline P1 & $26,333434 \mathrm{~S} ; 48,839770 \mathrm{~W} / 0,50$ & $25 / 12 / 2013$ & $24 / 03 / 2015$ & 292 & 81,95 & 174,01 & $1 \mathrm{E}-17$ \\
\hline S2 & $26,242627 \mathrm{~S} ; 48,802850 \mathrm{~W} / 0,32$ & $25 / 05 / 2010$ & $24 / 07 / 2011$ & 50 & 2,76 & 98,3 & $1 \mathrm{E}-17$ \\
\hline S3 & $26,305024 \mathrm{~S} ; 48,827940 \mathrm{~W} / 0,49$ & $25 / 04 / 2016$ & $24 / 12 / 2017$ & 132 & 41,46 & 64,6 & $1 \mathrm{E}-17$ \\
\hline S4 & $26,340529 \mathrm{~S} ; 48,851849 \mathrm{~W} / 0,41$ & $25 / 08 / 2012$ & $24 / 01 / 2014$ & 33 & 2,73 & 52,2 & $1 \mathrm{E}-17$ \\
\hline S5 & $26,263787 \mathrm{~S} ; 48,865306 \mathrm{~W} / 0,50$ & $25 / 03 / 2009$ & $24 / 01 / 2010$ & 24 & 1,73 & 41 & $1 \mathrm{E}-15$ \\
\hline S6 & $26,231720 \mathrm{~S} ; 48,831076 \mathrm{~W} / 0,45$ & $25 / 01 / 2017$ & $24 / 03 / 2017$ & 19 & 1,05 & 37,2 & $6 \mathrm{E}-14$ \\
\hline S7 & $26,363298 \mathrm{~S} ; 48,836680 \mathrm{~W} / 0,44$ & $25 / 01 / 2012$ & $24 / 03 / 2012$ & 14 & 0,47 & 33,9 & $2 \mathrm{E}-12$ \\
\hline S8 & $26,298037 \mathrm{~S} ; 48,896312 \mathrm{~W} / 0,29$ & $25 / 03 / 2014$ & $24 / 09 / 2014$ & 17 & 0,98 & 32,6 & $8 \mathrm{E}-12$ \\
\hline S9 & $26,300782 \mathrm{~S} ; 48,816093 \mathrm{~W} / 0,47$ & $25 / 12 / 2017$ & $24 / 09 / 2018$ & 78 & 28,05 & 30,5 & $8 \mathrm{E}-11$ \\
\hline S10 & $26,316942 \mathrm{~S} ; 48,817553 \mathrm{~W} / 0,47$ & $25 / 04 / 2017$ & $24 / 06 / 2018$ & 60 & 20,35 & 25,7 & 2E-08 \\
\hline S11 & $26,331139 \mathrm{~S} ; 48,814622 \mathrm{~W} / 0,35$ & $25 / 01 / 2018$ & $24 / 03 / 2018$ & 16 & 1,51 & 23,3 & 2E-07 \\
\hline S12 & $26,364069 \mathrm{~S} ; 48,822048 \mathrm{~W} / 0,40$ & $25 / 03 / 2012$ & $24 / 04 / 2012$ & 7 & 0,1 & 23,2 & 2E-07 \\
\hline S13 & $26,248765 \mathrm{~S} ; 48,906779 \mathrm{~W} / 0,39$ & $25 / 11 / 2009$ & $24 / 04 / 2011$ & 16 & 1,78 & 21 & $3 \mathrm{E}-06$ \\
\hline S14 & $26,230708 \mathrm{~S} ; 48,903360 \mathrm{~W} / 0,39$ & $25 / 11 / 2015$ & $24 / 01 / 2016$ & 7 & 0,14 & 20,8 & $3 \mathrm{E}-06$ \\
\hline S15 & $26,237473 \mathrm{~S} ; 48,809892 \mathrm{~W} / 0,22$ & 25/09/2017 & $24 / 10 / 2017$ & 5 & 0,04 & 19,8 & 9E-06 \\
\hline S16 & $26,252422 \mathrm{~S} ; 48,814817 \mathrm{~W} / 0,47$ & $25 / 11 / 2016$ & $24 / 12 / 2016$ & 5 & 0,04 & 19,3 & $2 \mathrm{E}-05$ \\
\hline S17 & $26,244589 \mathrm{~S} ; 48,819597 \mathrm{~W} / 0,06$ & $25 / 02 / 2012$ & $24 / 03 / 2012$ & 5 & 0,05 & 18,5 & $4 \mathrm{E}-05$ \\
\hline S18 & $26,297773 \mathrm{~S} ; 48,847501 \mathrm{~W} / 0,15$ & $25 / 01 / 2011$ & $24 / 02 / 2011$ & 4 & 0,02 & 16,9 & $2 \mathrm{E}-04$ \\
\hline S19 & $26,214755 \mathrm{~S} ; 48,900534 \mathrm{~W} / 0,25$ & $25 / 10 / 2009$ & $24 / 11 / 2009$ & 4 & 0,03 & 16 & $5 \mathrm{E}-04$ \\
\hline S20 & $26,259522 \mathrm{~S} ; 48,807487 \mathrm{~W} / 0,41$ & $25 / 01 / 2017$ & $24 / 05 / 2017$ & 8 & 0,56 & 13,8 & 0,01 \\
\hline S21 & $26,310058 \mathrm{~S} ; 48,842728 \mathrm{~W} / 0,33$ & $25 / 04 / 2018$ & $24 / 05 / 2018$ & 8 & 0,61 & 13,3 & 0,01 \\
\hline S22 & $26,340831 \mathrm{~S} ; 48,823405 \mathrm{~W} / 0,15$ & $25 / 02 / 2014$ & $24 / 05 / 2014$ & 7 & 0,42 & 13,2 & 0,01 \\
\hline S23 & $26,341858 \mathrm{~S} ; 48,831029 \mathrm{~W} / 0,45$ & $25 / 01 / 2013$ & $24 / 05 / 2013$ & 6 & 0,3 & 12,3 & 0,03 \\
\hline S24 & $26,211771 \mathrm{~S} ; 48,814186 \mathrm{~W} / 0,22$ & $25 / 03 / 2017$ & $24 / 04 / 2017$ & 4 & 0,07 & 12,3 & 0,03 \\
\hline S25 & $26,319863 \mathrm{~S} ; 48,875005 \mathrm{~W} / 0,03$ & $25 / 02 / 2015$ & $24 / 07 / 2015$ & 5 & 0,18 & 11,9 & 0,04 \\
\hline S26 & $26,324407 \mathrm{~S} ; 48,861952 \mathrm{~W} / 0,15$ & $25 / 02 / 2009$ & $24 / 06 / 2009$ & 3 & 0,02 & 11,9 & 0,04 \\
\hline
\end{tabular}

Fonte: Os autores (2021).

O único aglomerado que permaneceu ativo até o fim da pesquisa foi o secundário S9, que possui um raio de $0,47 \mathrm{Km}$ e centroide com latitude $26,300782^{\circ} \mathrm{S}$ e longitude $48,816093^{\circ} \mathrm{W}$, o qual está situado no bairro Boa Vista (Figura 5). O agrupamento iniciou em 25/12/2017 e perpetuou sua atividade até 24/09/2018, durante esses 9 meses foram observados 78 focos do vetor. Para aceitação da hipótese nula seriam esperados apenas 28,05 casos. É importante destacar que o aglomerado secundário S3 também pertence ao bairro Boa vista e sua atividade encerrou no dia 24/12/2017, próximo da data de início e da localização do cluster ativo. Isso pode ser um indicativo de uma migração do vetor no bairro ou uma nova infestação com outro meio de propagação. 
Figura 5 - Carta dos aglomerados de Ae. aegypti detectados pela análise retrospectiva em Joinville-SC no período de janeiro de 2009 a setembro de 2018.

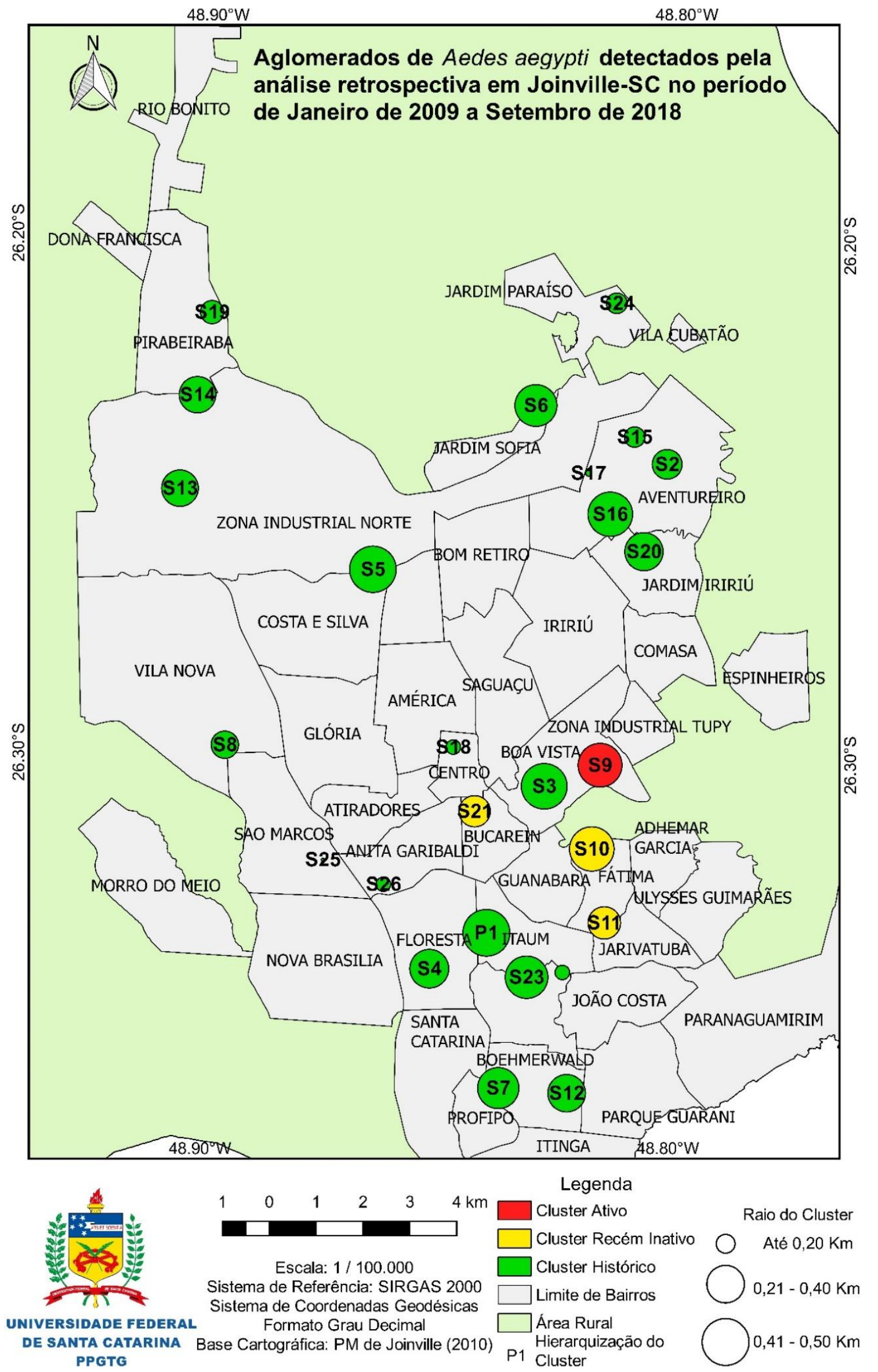

Fonte: Os autores (2021). 
Três aglomerados se tornaram históricos em 2018, isto é, próximo ao fim do período do estudo. O secundário S10 abrangeu um raio de $0,47 \mathrm{Km}$ a partir do centroide de latitude $26,316942^{\circ} \mathrm{S}$ e longitude $48,817553^{\circ} \mathrm{W}$, localizado no bairro Fátima. Este conglomerado permaneceu ativo durante 14 meses, de 25/04/2017 a 24/06/2018, contemplou 60 criadouros contra 20,35 sob a hipótese nula. O secundário S11 também foi detectado nesse bairro com um raio de $0,35 \mathrm{Km}$ e centroide com latitude $26,331139^{\circ} \mathrm{S}$ e longitude $48,814622^{\circ} \mathrm{W}$. Persistiu de 25/01/2018 até 24/03/2018 e contou com 16 focos do mosquito, sendo que pela hipótese nula seriam esperados 1,51 casos. Pelas datas de início e fim dos mesmos constatou-se que durante um determinado período de 2018 houve a existência concomitante dos dois no bairro, o secundário S10 localizado na fronteira norte e o secundário S11 na Sul (Figura 5).

O último cluster recém inativo até o fim do experimento foi o secundário S21, o único existente no bairro Bucarein, que teve um raio de $0,33 \mathrm{Km}$ e centroide de latitude $26,310058^{\circ} \mathrm{S}$ e longitude $48,842728^{\circ} \mathrm{W}$. Entre 25/04/2018 a 24/05/2018 eram esperados apenas 0,61 casos para aceitação da hipótese nula, porém foram notificados 8 focos de Ae. aegypti.

Do ponto de vista espacial notou-se que esses quatro clusters (ativo e recém inativos) estavam estabelecidos próximos, por exemplo, o bairro Boa Vista e Bucarein que tem limites comuns. Isso demonstra que a infestação estava contida em uma pequena porção do território. Os demais agrupamentos foram históricos.

Analisando que a zona urbana municipal de Joinville abrange uma área de 21.056,65 ha, a infestação (ativa e recém inativa) estava restrita a 210,73 ha, o que corresponde a 1,00\% do território urbano. Supondo que o poder público realizasse uma ação para a eliminação de focos em áreas precárias, o monitoramento pela hierarquização dos aglomerados se tornaria mais viável devido a qualidade do serviço e o número de pessoas envolvidas na prática.

Em relação à evolução temporal verificou-se que em todos os anos houve o início de novos conglomerados. No ano de 2017 o número foi de 6 clusters, ou seja, a maior quantidade em um ano entre a série analisada (Tabela 2). Na sequência, tem-se 2009 e 2012 com 4 detecções. As menores quantidades ocorreram nos anos de 2010 e 2011 com a formação de um único agrupamento em cada.

A duração variou entre 1 a 20 meses, sendo que a máxima foi observada no agrupamento secundário S3. Já o período mínimo foi detectado em 8 conglomerados, por exemplo, nos secundários S12 e S15. Com isso, percebe-se que a sazonalidade no número de focos do inseto, apontada no Gráfico 1, não interferiu na formação e na manutenção dos agrupamentos do vetor.

Ainda assim, é possível que essa sazonalidade dos focos do vetor tenha relação com a co-variável precipitação total mensal, cujo estudo de Merêncio (2019) identificou que essa co-variável foi responsável pela origem de 51,85\% dos agrupamentos de Ae. aegypti detectados entre janeiro de 2009 e setembro de 2018 em Joinville. Entretanto, o autor destacou a importância da aplicação da estatística Scan no controle do vetor sem o uso de co-variáveis, em virtude da elevada quantidade de clusters detectados sem explicação da precipitação e dos diversos fatores ambientais e sociais que podem estar associados ao desenvolvimento do inseto (MENDES, 2016).

Os resultados apresentados até aqui permitem constatar que o modelo de permutação espaço-temporal é capaz de determinar a distribuição dos conglomerados de Ae. aegypti no espaço e no tempo, visto que nos bairros com mais focos ocorreu a detecção de clusters, como no bairro Boa Vista, que é o primeiro em notificações e possui dois agrupamentos, $S 3$ e S9. Este último foi o único ativo na área de estudo até setembro de 2018.

Considerando o contexto da infestação no município de Joinville entre janeiro de 2009 e setembro de 2018 destaca-se que a utilização da estatística Scan determinou que os conglomerados de Ae. aegypti na cidade são significativos ao nível de 5\%, tanto os históricos quanto os ativos.

Nesse contexto, verificou-se a importância das cartas como instrumento de visualização científica em razão da sua capacidade de representar os conglomerados do Ae. aegypti, isto é, as áreas onde a concentração do fenômeno foi mais intensa (SILVA, 2013).

O uso da estatística Scan para o estudo da evolução espaço-temporal do mosquito demonstrou-se útil, pois restringiu e hierarquizou agrupamentos na área de varredura, tornando a atividade de vigilância mais 
viável, comparada ao tamanho da zona urbana municipal. Ainda, identificou o início e a extinção dos clusters nos bairros.

Segata (2018) questionou a aplicabilidade das tecnologias digitais e seus benefícios no combate ao vetor, afirmando que a utilização de softwares com esta finalidade produz periferias morais e geográficas que segregam o espaço municipal, e que as relações humano-animal devem ser respeitadas para o desenvolvimento de estratégias de controle. Em contrapartida, Barbosa et al. (2017) atestou que o uso da estatística Scan em Natal-RN minimizou os impactos da epidemia de 2015, já que o número de casos notificados foi menor que o das epidemias anteriores. Na prática, a utilização da estatística Scan em Joinville mostrou que os parâmetros espaciais e temporais são maleáveis no SATSCAN 9.6, e permitem a inclusão de co-variáveis diversas, buscando gerar resultados próximos da realidade local. Assim, o método deve ser utilizado para nortear a tomada de decisões, associado a outros campos do conhecimento e observando o contexto da infestação.

\section{CONCLUSÃO}

A necessidade do aperfeiçoamento dos métodos de controle de Ae. aegypti aplicados em Joinville é uma questão que demanda atenção, pois houve o aumento do número de focos registrados no decorrer dos anos. Nesse sentido, este artigo atingiu o objetivo de aplicar a estatística de varredura para a identificação dos conglomerados deste vetor na cidade ao nível de significância de 5\%, hierarquizando-os conforme a prioridade de intervenção.

Pela análise retrospectiva delimitou-se 26 conglomerados na zona urbana de Joinville entre janeiro de 2009 a setembro de 2018. Apenas 1 permaneceu ativo até o término do estudo. Esse agrupamento localizouse no bairro Boa vista, o que evidencia a necessidade de ações dos gestores públicos para reverter essa situação.

A estatística de varredura de Kulldorff et al. (2005) é uma técnica de baixo custo, não necessita de recursos humanos e materiais em excesso e utiliza dados de fácil obtenção ou até que já compõem a série de dados municipal. Portanto, é um procedimento aplicável a todas as cidades catarinenses, sendo adaptável conforme as particularidades de cada município, ampliando assim, a sua atuação para outros estados.

Verificou-se que os resultados do método são sensíveis as configurações informadas no software, por isso, é essencial que antes da execução essas configurações estejam bem definidas e apoiadas na literatura, pois a delimitação dessas por tentativas podem gerar resultados incertos.

Para trabalhos futuros recomenda-se a inclusão de co-variáveis no modelo espaço-temporal e a verificação da influência dessas nos aglomerados para compreender o contexto geral. Dentre os diversos fatores envolvidos, seria interessante avaliar o efeito da temperatura média do ar nos índices de infestação. A introdução de dados socioeconômicos na estatística também é um ponto a ser considerado, já que possibilitaria a análise dos surtos relacionada às questões sociais da população e suas habitações. Outro estudo pertinente seria a utilização prática da estatística Scan, com o monitoramento da expansão dos focos em campo baseado na hierarquização dos clusters e, na sequência, comparar os resultados do método com as séries de focos anteriores.

\section{Agradecimentos}

Os autores agradecem à Diretoria de Vigilância Epidemiológica de Santa Catarina (DIVE-SC) pelo fornecimento dos registros referentes ao Aedes aegypti na cidade de Joinville.

\section{Contribuição dos Autores}

O autor Ivan Merêncio foi responsável pela concepção da ideia do artigo, curadoria dos dados, metodologia, análise formal, redação da minuta inicial, revisão e edição. O autor Carlos Antonio Oliveira Vieira executou a análise formal, a supervisão, a revisão e edição deste artigo.

\section{Conflitos de Interesse}


Os autores declaram que não há conflitos de interesse.

\section{Referências}

ARAÚJO, T. C. Extensão da estatística Scan para detecção de conglomerados espaço-temporais em dados com excesso de zeros. 2012. 89 f. Dissertação (Mestrado) - Curso de Estatística, UNB, Brasília, 2012. Disponível em: <http://repositorio.unb.br/handle/10482/12804>. Acesso em: 13 fev. 2019.

AYUBI, E. et al. Spatial Modeling of Cutaneous Leishmaniasis in Iranian Army Units During 2014-2017 Using a Hierarchical Bayesian Method and the Spatial Scan Statistic. Epidemiology And Health, [s.1.], v. 40, p.1-9, 13 jul. 2018. DOI. 10.4178/epih.e2018032

BALIEIRO, A. A. da. S. et al. O Uso do Sensoriamento Remoto e da Estatística de Varredura (Scan) na Detecção e Quantificação em Significância de Agrupamentos de Desmatamento no Sul da Amazônia. In: Simpósio Brasileiro de Sensoriamento Remoto - SBSR, 14, 2009, Natal. Anais... Natal, 2009. Disponível em: <http://marte.sid.inpe.br/col/dpi.inpe.br/sbsr@80/2008/11.17.22. 40.35/doc/5359-5365.pdf>. Acesso em: 16 fev. 2019.

BALIEIRO, A. A. S. Detecção de conglomerados dos alertas de desmatamentos no Estado do Amazonas usando estatística de varredura espaço-temporal. 2008. 87 f. Dissertação (Mestrado em Estatística Aplicada e Biometria) - Universidade Federal de Viçosa, Viçosa, MG, 2008.

BARBOSA, A. Estudos Preliminares sobre o Campo Térmico de Joinville - SC. 2009.87 f. Tese (Doutorado) - Curso de Geografia, Centro de Filosofia e Ciências Humanas, Universidade Federal de Santa Catarina, Joinville, 2009.

BARBOSA, I. R. et al. Identificação de áreas prioritárias para a vigilância e controle de dengue e outras arboviroses transmitidas pelo Aedes aegypti no município de Natal-RN: relato de experiência. Epidemiologia e Serviços de Saúde, [s.1.], v. 26, n. 3, p.629-638, jul. 2017. DOI. 10.5123/s167949742017000300020.

BRASIL. Casos de Dengue. Brasil, Grandes Regiões e Unidades Federadas. 1990 a 2016. 2017. Disponível em: <http://portalarquivos.saude.gov.br/images/pdf/2017/fevereiro/10/Dengue-classica-ate-2016.pdf> Acesso em: 16 ago. 2018.

BUSATO, M. A et al. Evolução da Infestação por Aedes aegypti (Diptera: Culicidae) nos Municípios do Oeste do Estado de Santa Catarina. Saúde Pública SC, Florianópolis, v. 7, n. 2, p.107-118, ago. 2014. Disponível em: <https://www.unochapeco.edu.br/static/data/portal/downloads/2739.pdf>. Acesso em: 19 fev. 2019.

DICK, Olivia Brathwaite et al. The History of Dengue Outbreaks in the Americas. The American Journal Of Tropical Medicine And Hygiene, [s.1.], v. 87, n. 4, p.584-593, 3 out. 2012. DOI. 10.4269/ajtmh.2012.110770 .

FREITAS, R. M.; OLIVEIRA, R. L. Presumed unconstrained dispersal of Aedes aegypti in the city of Rio de Janeiro. Brasil, Revista de Saúde Pública. São Paulo, v. 1, n. 43, p. 8-12, 2009.

GOMES, R. G. S. Epidemiologia das Cardiopatias Congênitas e a Influência de Fatores SócioAmbientais no Estado da Paraíba. 2016. 96 f. Tese (Doutorado) - Curso de Biologia Aplicada à Saúde, UFPE, Recife, 2016. Disponível em: <https://repositorio.ufpe.br/handle/123456789/28083>. Acesso em: 15 jan. 2019.

HELBICH, M; LEITNER, M. Evaluation of Spatial Cluster Detection Algorithms for Crime Locations. Challenges At The Interface Of Data Analysis, Computer Science, And Optimization, [s.1.], p.193201, 2012. Springer Berlin Heidelberg. DOI. 10.1007/978-3-642-24466-7_20

INSTITUTO BRASILEIRO DE GEOGRAFIA E ESTATÍSTICA - IBGE. IBGE Cidades: Território e Ambiente. 2017. Disponível em: <https://cidades.ibge.gov.br/brasil/sc/joinville/panorama>. Acesso em: 26 ago. 2018.

JOINVILlE. Diagnóstico Socioambiental. 2. ed. Joinville: PMJ, 2016. 155 p. Disponível em: 
<https://www.joinville.sc.gov.br/wp-content/uploads/2016/07/\%C3\%81rea-urbana-consolidada-deJoinville-Volume-II-Diagn\%C3\%B3stico-socioambiental.pdf>. Acesso em: 15 ago. 2018.

JOINVILLE. Secretaria de Planejamento Urbano e Desenvolvimento Sustentável. Joinville Cidade em Dados 2017. Joinville, 2017. 73 p. Disponível em: <https://www.joinville.sc.gov.br/wpcontent/uploads/2016/01/Joinville-Cidade-em-Dados-2017.pdf>. Acesso em: 15 ago. 2018.

JUNG, I. et al. A Spatial Scan Statistic for Multinomial Data. Statistics In Medicine, [s.1.], v. 29, n. 18, p.19101918, maio 2010. DOI. 10.1002/sim.3951.

KAQUI, R. L. Uma Estatística de Varredura Espacial para Dados de Contagem com Censura. 2016. 98

f. Dissertação (Mestrado) - Curso de Estatística, UnB, Brasília, 2016. Disponível em: <http://repositorio.unb.br/bitstream/10482/23067/3/2016_RobertoLazarte Kaqui.pdf>. Acesso em: 20 jan. 2019.

KRAEMER, M. U. G. et al. The global compendium of Aedes aegypti and Ae. albopictus occurrence. Scientific Data, [s.1.], v. 2, p.1-8, 7 jul. 2015. DOI. 10.1038/sdata.2015.35.

KULlDORFF, M. et al. A Space-Time Permutation Scan Statistic for Disease Outbreak Detection. Plos Medicine, [s.1.], v. 2, n. 3, p.216-224, fev. 2005. DOI. 10.1371/journal.pmed.0020059.

KULLDORFF, M. et al. Evaluating cluster alarms: A space-time scan statistic and brain cancer in Los Alamos. American Journal of Public Health, [s.1.], v. 88, n. 9, p.1377-1380, 1998.

KULLDORFF, M. SatScanTM user guide. 2018. Disponível em: <https://www.satscan.org/cgibin/satscan/register.pl/SaTScan_Users_Guide.pdf?todo=process_userguide_download $>$. Acesso em: 31 de julho de 2018.

KULLDORFF, M.; NAGARWALLA, N. Spatial disease cluster: detection and inference. Statistics in Medicine. v. 14, p. 799-810. 1995

MATEUS, A. L. S. S. Proposição de Novas Metodologias para Análise de Aleatoriedade em Processos Pontuais no Espaço-Tempo. 2013. 138 f. Tese (Doutorado) - Curso de Estatística e Experimentação Agropecuária, Universidade Federal de Lavras, Lavras, 2013.

MENDES, J. A. Influência do Padrão Espacial do Uso do Solo Urbano e da Proximidade de Criadouros do Aedes aegypti na Ocorrência de Casos de Dengue Durante a Epidemia de 2013 em Campinas- Sp. 2016. 81 f. Dissertação (Mestrado) - Curso de Geografia, UNICAMP, Campinas, 2016. Disponível em: <http://repositorio.unicamp.br/jspui/handle/REPOSIP/330405>. Acesso em: 16 jan. 2019.

MERÊNCIO, I. Detecção de conglomerados de focos de Aedes aegypti em Joinville através da estatística de varredura espaço-temporal. 2019. 128 f. Dissertação (Mestrado) - Curso de Engenharia de Transportes e Gestão Territorial, Centro Tecnológico, Universidade Federal de Santa Catarina - UFSC, Florianópolis, 2019.

OLIVEIRA, F. A. de. Estudo do aporte sedimentar em suspensão na baía da babitonga sob a ótica da geomorfologia. Tese (Doutor em Geografia) - Universidade de São Paulo - USP. Faculdade de Filosofia, Letras e Ciências Humanas, 2006

ORGANIZAÇÃO PANAMERICANA DE SAÚDE (OPAS). Dengue: guías para el diagnostico, tratamiento, prevención y control. La Paz: Bolívia, 2010.

RIBEIRO, M. D. et al. Estudo descritivo da ocorrência de dengue e sua relação com o clima e a ação da vigilância em saúde do município de Franca, São Paulo, Brasil, 2007 a 2011. Revista Investigação Saúde, Franca, v. 14, n. 1, p. 138-144, 2015. Disponível em: < http://publicacoes.unifran.br/index.php/investigacao/article/view/737>. Acesso em: 19 fev. 2019.

SANTA CATARINA. DIRETORIA DE VIGILÂNCIA EPIDEMIOLÓGICA (DIVE). Boletim Epidemiológico $\quad \mathbf{n}^{\circ}$ 12/2017. Florianópolis: DIVE, 2017b. Disponível em: <http://www.dive.sc.gov.br/index.php/arquivo-noticias/634-boletim-epidemiologico-n-26-2017vigilancia-entomologica-do-aedes-aegypti-e-situacao-epidemiologica-da-dengue-febre-de-chikungunyae-zika-virus-em-santa-catarina-atualizado-em-30-12-2017-se-52-2017>. Acesso em: 20 ago. 2018. 
. DIRETORIA DE VIGILÂNCIA EPIDEMIOLÓGICA (DIVE). Programa de Controle de Dengue. Florianópolis: DIVE, 2017a. Disponível

SANTANA, N. A. de. A Produção do Espaço Urbano e os Loteamentos na Cidade de Joinville (SC) 1949-1996. Florianópolis. UFSC. Departamento de Geociências, 1998. (Dissertação de Mestrado).

SEGATA, J. Infraestruturas globais, práticas locais: o Aedes aegypti e o digital. In: SEGATA, J.; RIFIOTIS,

T. Políticas etnográficas no campo da ciência e das tecnologias da vida. Porto Alegre: UFRGS, 2018. p. 61-84.

SILVA, C. N. da. A Representação Espacial e a Linguagem Cartográfica. Belém: ED. Gapta/UFPA, 2013. $182 \mathrm{p}$.

SILVA, S. B. da. Análise de Fronteiras Geográficas e Limites no Processo Temporal para Avaliação de Conglomerados Espaço-Temporais. 2016. 78 f. Tese (Doutorado) - Curso de Estatística, UFMG, Belo Horizonte, 2016. Disponível em: <http://www.bibliotecadigital.ufmg.br/dspace/handle/1843/BUBDAA2FH9>. Acesso em: 20 jan. 2019.

\section{Biografia do autor principal}

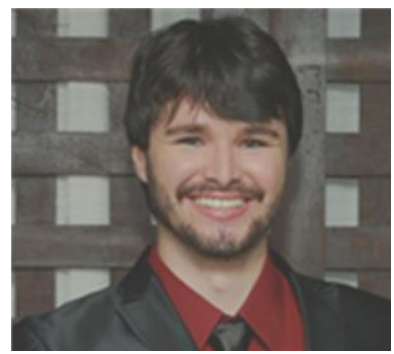

Ivan Merêncio, natural de Meleiro, SC, Brasil. Possui graduação em Engenharia de Agrimensura pela Universidade do Extremo Sul Catarinense (2016) e mestrado em Engenharia de Transportes e Gestão Territorial pela Universidade Federal de Santa Catarina (2019). Atualmente trabalha como Engenheiro Agrimensor em uma empresa do setor privado de Meleiro. Tem experiência na área de Geociências, com ênfase em Cadastro Territorial Multifinalitário (CTM), Sistema de Informação Geográfica (SIG), Estatística Espacial Scan, Cartografia, Topografia e Geodésia. 